\title{
Taratura di un'apparecchiatura per misura di piccole percentuali di uranio in soluzione acida di acqua distillata
}

\author{
M. DIANA
}

Ricevuto il 30 Gennaio 1965

\begin{abstract}
Riassunto. - Si descrive una tecnica che permette di misurare il numero di atomi di Uranio di una soluzione, con un errore non superiore al $10 \%$.

Il sistema consiste in un tubo di Geiger (Plilips 18503) sistemato in un contenitore di vetro nel quale viene diluita una certa quantità di peso di nitrato di Uranile, misurato con una precisione migliore dell' $1 \%$. $\Lambda \mathrm{l}$ variare della diluizione si registra il numero di particelle che il Geiger conta, e quindi si riporta in grafico. Da questi per diverse quantità di sale sciolto si determina la retta di taratura.
\end{abstract}

SUMMary. - A technique allowing to measure the Uranium atoms number of a solution with an error smaller than $10 \%$ is described.

The apparatus consists of a Geiger tube (Philips 18503) enclosed in a glass wessel. In this wessel is diluted a certain weight of Uranyl nitrate. The weight of sample is known with a precision better than $1 \%$. The particles number detected by the Geiger tube, versus dilution, is plotted. From these curves plotted for samples of different weight, one determines the calibration straight line.

\section{1. - INTRODUZIONE.}

Un notevole contributo allo studio della sezione d'urto per fotofissione dell'Uranio in vicinanza della soglia, è stato dato dalla nuova tecnica di caricamente e sviluppo delle emulsioni nucleari (1, “). Il caricamento delle emulsioni con Uranio si ottiene mescolando all'emulsione nucleare in forma di gel una quantità nota di citrato di Uranio di composizione perfettamente nota. La mistura viene poi diluita con acqua, 
sciolta e versata in un opportuno recipiente dove viene seccata. Si forma così una pellicola facilmente staccabile e di spessore piuttosto uniforme. Queste pellicole vengono poi esposte ad un flusso noto di raggi gamma monocromatici, e dopo opportuno sviluppo per eliminare le tracce di particelle alfa $\left(^{1}\right)$ vengono esaminate al microscopio. Se $N_{f}$ è il numero di fissioni trovato nella pellicola, la sezione d'urto per fotofissione è data da

$$
\sigma_{f}=\frac{N_{f}}{\varphi N}
$$

dove $N$ è il numero dei nuclei di Uranio nella pellicola, e $\varphi$ il flusso di gamma. La determinazione di $N$ non è così semplice come sembra in quanto non si conosce mai con esattezza quanto citrato di Uranio è rimasto nei recipienti in cui l'emulsione è stata sciolta e seccata, né si è sicuri che l'Uranio si distribuisca uniformemente nella pellicola. Si sono studiati allora vari metodi per cercare di determinare $N$. Uno di questi consiste nel lavare la pellicola in un ciclo chiuso facendo evaporare dell'acqua distillata e facendola poi condensare in un recipiente dove è stata posta la pellicola.

Da precedenti esperienze si è visto che due ore di lavaggio portano praticamente via tutto l'Uranio della pellicola. Si tratta ora di determinare la concentrazione in Uranio della soluzione che si è ottenuta.

L'errore che si richiede nella determinazione di $N$ deve essere entro il $10 \%$, con la conseguenza che la sezione d'urto viene data con la stessa precisione. Il presente lavoro quindi si propone di elaborare una tecnica che permetta di misurare $N$ entro i limiti di errori anzidetti. Per questo tutte le quantità di Uranio che venivano sciolte in soluzione erano date con la precisione di $\pm 0,2 \mathrm{mg}$.

\section{2. - Determinazione della QuaNtità di URanio Nelle soluzioni DI LAVAGGIO.}

Il metodo seguito è quello di diluire una certa quantità (in peso) di nitrato di Uranile $\left(\mathrm{UO}_{2}\left(\mathrm{NO}_{3}\right)_{2}+6 \mathrm{H}_{2} \mathrm{O}\right)$ e registrare il valore del conteggio alle varie diluizioni. Inconveniente principale da eliminare è la proprietà dell'Uranio di venire adsorbito dalle parti dell'apparechiatura con le quali è a contatto. Per ovviare a questo si sono dovute usare soluzioni acide con $\mathrm{pH}$ minore od uguale ad 1. Per ottenere le quali sono bastate 10-15 goce di $\mathrm{HCl}$ commerciale aggiunti in ogni soluzione. 
Lo schema a blocchi dell'apparecchiatura usata ì il seguente:

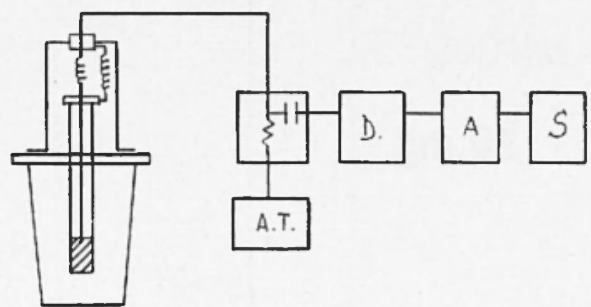

Fig. 1.

Il tubo di Geiger usato era un 18503 della Philips adatto per conteggi di particelle gamma, questo Geiger era sistemato sul fondo di una provetta di vetro ehiusa all'estremità superiore da plexiglass attraverso il quale passavano i collegamenti (Fig. 2).

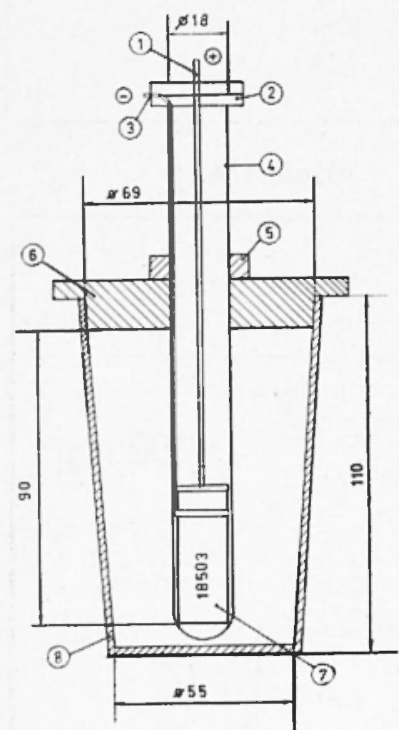

Fig. 2.

1) Filo rigido (positivo) di rame argentato dello spessore di $2 \mathrm{~mm}$. - 2) Tappo di chiusura (plexiglass) della provetta. - 3) Striscetta di rame (negativo) di 2 decimi di millimetro di spessore. - 4) Provetta di vetro. - 5) Anello di plexiglass regolatore dell'altezza della provetta. - 6) Tappo di plexiglass che chiude il bicchiere. - 7) Contatore di Geiger tipo 18503 della Philips. - 8) Bicchiere di vetro contenente la soluzione.

Questa provetta veniva regolata in alteza attraverso un foro praticuto nel tappo del bicchiere. Il tutto era sistemato entro un pozzetto di piombo di $3 \mathrm{~cm}$ di spessore in modo da ridurre il più possibile la radiazione di fondo. Attraverso poi una scatola di partizione gli impulsi venivano contati da una scala con soglia di 5 Volt.

Ogni misura era preceduta da una di fondo per determinare le condizioni di lavoro relative ad ogni quantità di soluto. 

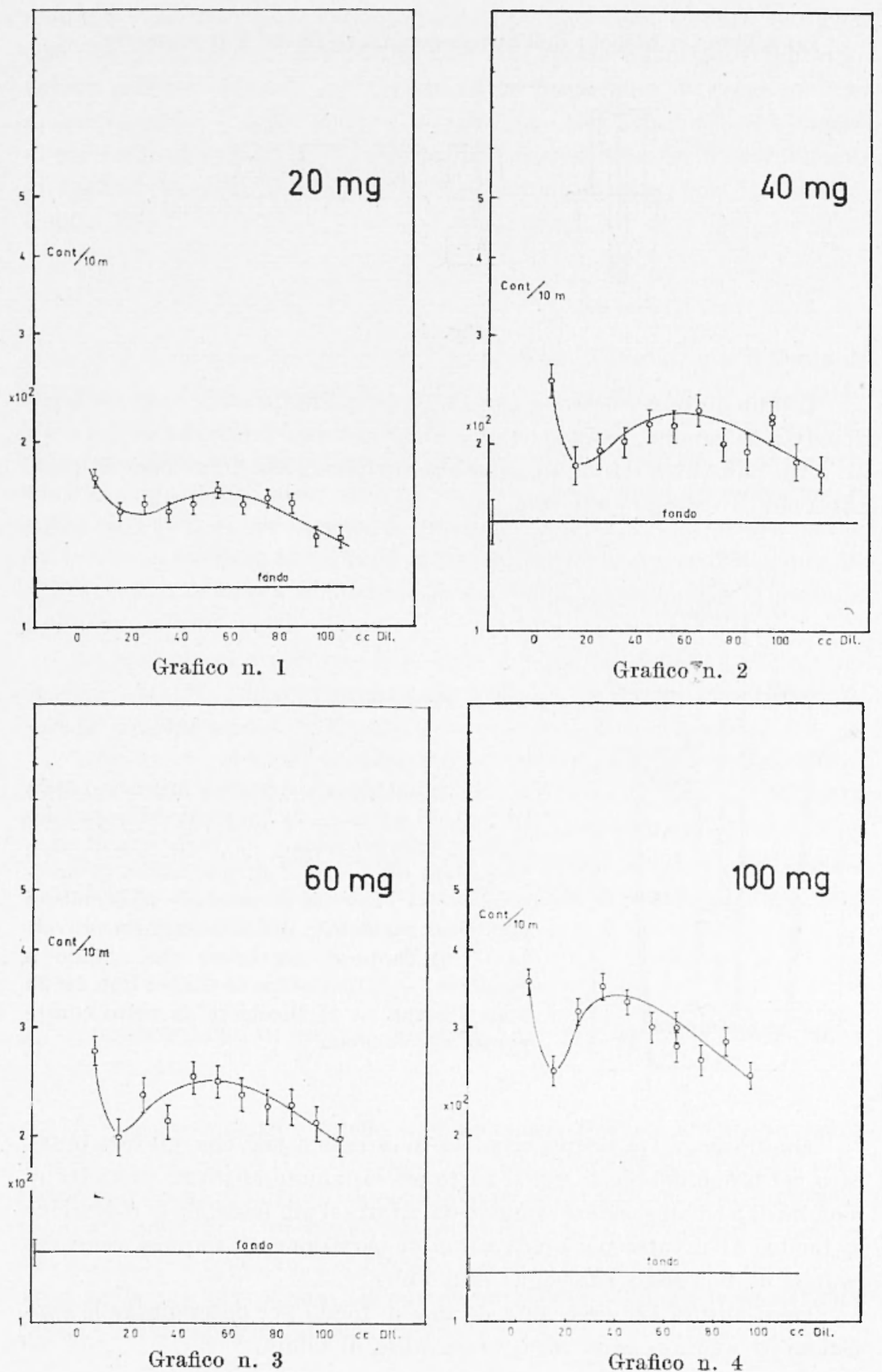


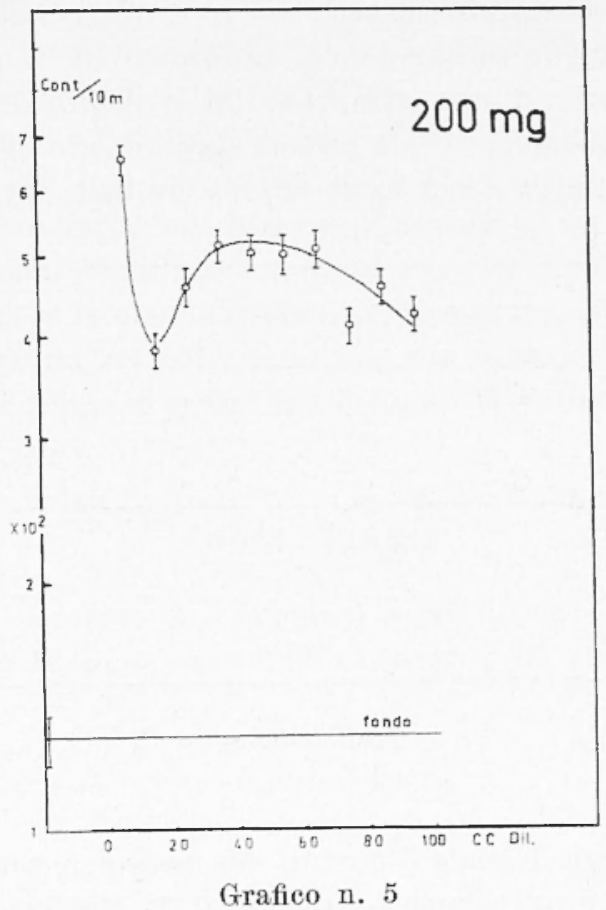

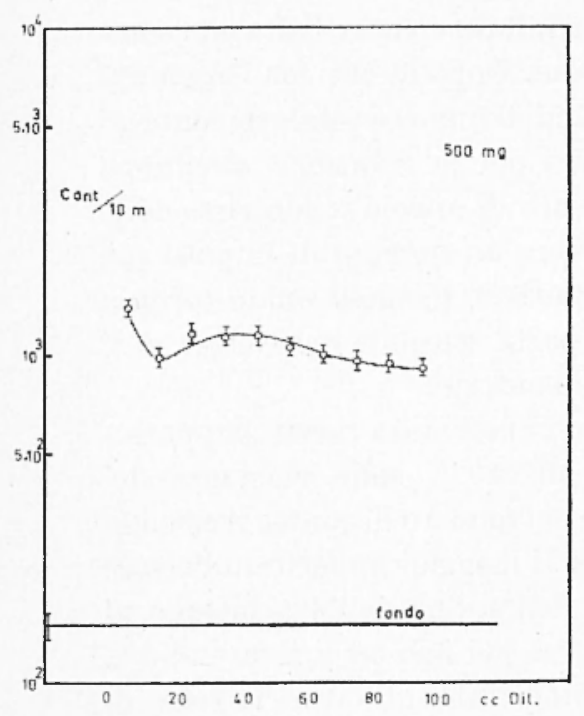

Grafico n. 6

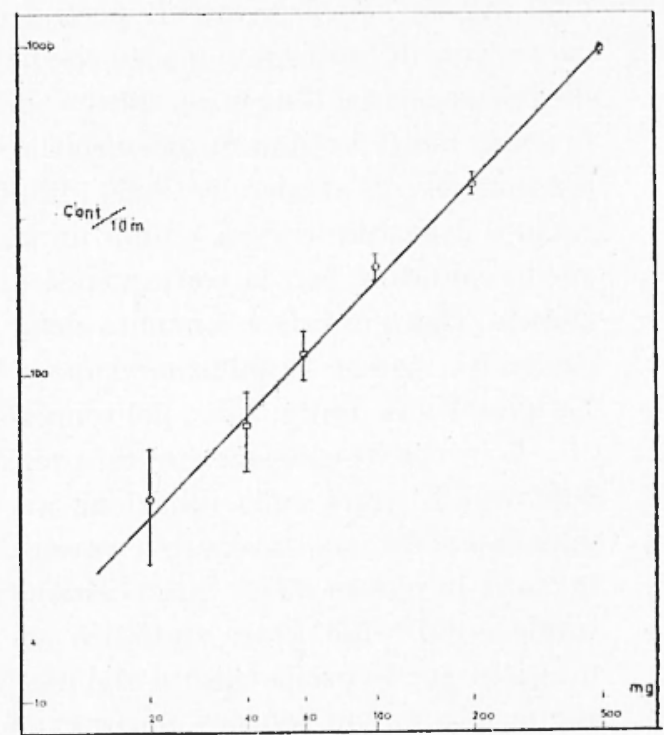

Grafico n. 7 
Importante constatare che tutte le curve, relative alla variazione del mumero di conteggi in funzione della diluizione con acqua distillata ed ognuma caratteristica di una certa quantità di nitrato di Uranile sciolta, hanno lo stesso andamento, con piccolo spostamento della posizione del massimo verso diluizioni più basse al crescere della quantità di soluto.

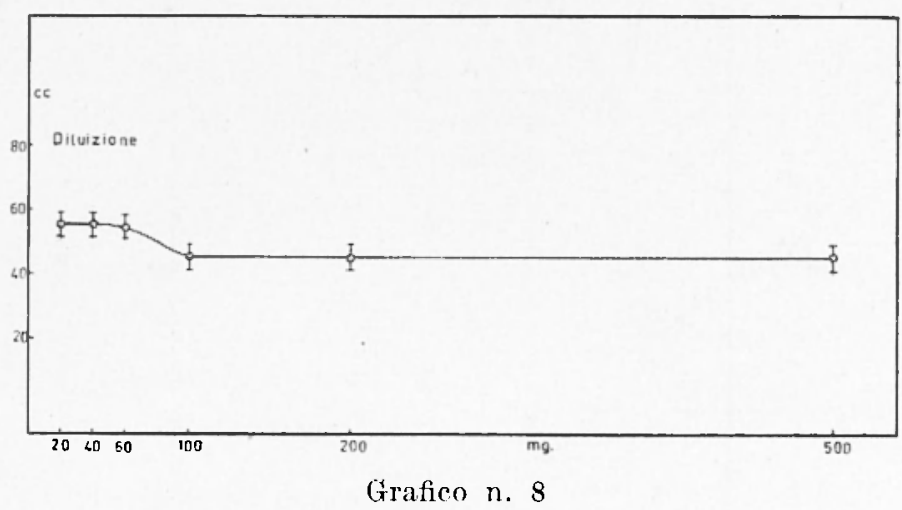

I'alto conteggio iniziale è dovuto alla preponderante influenza dei raggi beta emessi principalmente dall' $\mathrm{UX}_{2}(2,31 . \mathrm{MeV})$ sul numero totale di gamma. Questi raggi beta, per la sottilezza delle pareti del contatore $\left(250 \mathrm{mg} / \mathrm{cm}^{2}\right)$ in presenza di poca acqua, vengono facilmente contati; sul numero di conteggi in queste condizioni influisce anche l'alta efficienza di rivelazione del Geiger per dette radiazioni. Dopo di che con l'aggiunta di acqua molti vengono autoassorbiti e quindi il numero totale di conteggi dimimuisce. All'aumentare della diluizione poi la soluzione avviluppa tutto il contatore e si ha quindi un aumento di angolo solirlo visto dallo stesso contatore con la conseguenza di avere un numero di impulsi più elevato. Con l'ulteriore aumento della diluizione l'angolo solido tormal a dimimuire, perché la soluzione supera la parte sensibile del Geiger, e si ha quindi una diminuzione del numero di conteggi.

È in corrispondenza a questo massimo che è stata tamata l'apparecchiatura. Ijerrore sulla diluizione anche del 10\% nelle vicinanze del massimo porta una trascumbile variazione del numero di conteggi essendo la curva in questo punto quasi orizzontale. Il maggior apporto sull'errore totale è dato dall'errore statistico che è dell'ordine dell's\% intorno al massimo per le prime misure che diminuisce poi fino arl arrivare al $3 \%$ per la misura con $500 \mathrm{mg}$. Sommando quindi tutti gli errori la retta di taratura è data con una precisione di cirea il 10\%. 
Come si vede nel grafico no 7 la variazione del numero di impulsi al valore massimo anzidetto in funzione della quantità di Uranio sciolta nella soluzione è entro gli errori una retta.

Nel glafico n. 8 è poi riportata la variazione della posizione del massimo numero di conteggi in funzione della diluizione e della quantità di sale sciolto. Questa variazione può essere doruta ad un residuo di adsorbimento dell'Uranio più sensibile a basse quantità di soluto ed la quindi l'effetto di spostare il massimo verso diluizioni maggiori. Quest'ultimo grafico serve quando si debba misurare una quantita incognita di cui però si conosca l'ordine di grandezza del peso di prodotto attivo sciolto.

\section{BIBLIOGRAFIA}

(1) De Carvalifo H. G. et al., "Suppl. al Nuovo Cimento ", 19, 1131 (1961).

(2) De Carvalio II. G. et al.: "Nuovo Cimento", 25, 534 (1962). 